\title{
Voltage and current excitation for time-harmonic eddy-current problems
}

\author{
Ana Alonso Rodríguez * Alberto Valli *
}

\begin{abstract}
We want to give a systematic presentation of voltage or current intensity excitation for time-harmonic eddy-current problems. The key point of our approach resides in a suitable power law, that permits to understand the role of voltage excitation. We also enlighten the influence of the boundary conditions on the proposed formulations.
\end{abstract}

\section{Introduction and basic results}

In many electromagnetic phenomena it is useful to couple formulations in terms of electrical circuits with more general formulations based on the Maxwell equations (or else on some reduced model like the eddy-current system).

This coupling is often performed by transforming some data like voltage and current intensity into suitable boundary conditions for the electromagnetic fields. In particular, it is interesting to devise efficient formulations of the eddycurrent problem when the only present excitation is either an assigned voltage (typically, at contacts) or a given current intensity in the eddy-current region.

On the other hand, it is well-known that the topological properties of the conductor and the type of boundary conditions imposed on the boundary of the computational domain have a strong influence on the general setting of the problem and on the structure of the solution.

Several possible approaches have been proposed in the recent years, especially by engineers interested in practical computations. Far from being complete, let us only mention the contributions in [11], [19], [17], [18], [25], [26], [23], [16], [10], [20], [8], [6] and the references therein.

In this paper we propose a systematic approach to eddy-current problems driven by voltage or current intensity. Our aim is to give a general mathematical formulation for these problems, and to analyze their well-posedness. These theoretical results are then the basis for devising stable and convergent finite element approximation schemes.

A typical difficulty is that, in many situations, the eddy-current problems are well-posed even if no additional condition like voltage or current intensity

\footnotetext{
*Dipartimento di Matematica, Università di Trento, I-38050 Povo (Trento); alonso@science.unitn.it, valli@science.unitn.it
} 
is imposed. As a consequence, to overcome this apparent contradiction it is necessary to focus on the modelling of the problem, so that it becomes possible to impose the voltage or current intensity equation, but without renouncing to the Maxwell equations (a flaw that was present in previous papers on this subject).

In the rest of this Section we are introducing notation and describing the problems we consider, and we are presenting two basic results concerning wellposedness. In Section 2 we are discussing about modelling, basing our argument on a global power law that relates voltage to current intensity. In the third section our proposal for treating voltage and current excitation problems is described. In Section 4 we systematically present the variational formulations of these problems. Finally, the last section is devoted to give a short description of some numerical approximation schemes based on finite elements.

In the following, for the sake of simplicity we assume that the domain $\Omega \subset \mathbb{R}^{3}$ is a simply-connected bounded open set, with a connected boundary $\partial \Omega$. It is composed by two parts, a conductor $\Omega_{C}$ and an insulator $\Omega_{I}$. The interface between $\Omega_{C}$ and $\Omega_{I}$ will be denoted by $\Gamma$. The unit outward normal vector on $\partial \Omega$ will be indicated by $\mathbf{n}$, while the unit normal vector on $\Gamma$, directed towards $\Omega_{I}$, will be denoted by $\mathbf{n}_{C}=-\mathbf{n}_{I}$.

As it is well-known (see, e.g., [13]), the time-harmonic eddy-current problem is given by the Ampère and Faraday equations

$$
\begin{array}{ll}
\operatorname{curl} \mathbf{H}-\boldsymbol{\sigma} \mathbf{E}=\mathbf{J}_{e} & \text { in } \Omega \\
\operatorname{curl} \mathbf{E}+i \omega \boldsymbol{\mu} \mathbf{H}=\mathbf{0} & \text { in } \Omega,
\end{array}
$$

where $\mathbf{H}$ and $\mathbf{E}$ are the magnetic and electric field, respectively, $\mathbf{J}_{e}$ is the given electric current density, $\boldsymbol{\sigma}$ is the electric conductivity, $\boldsymbol{\mu}$ is the magnetic permeability, and $\omega \neq 0$ is a given angular frequency. Moreover, suitable boundary conditions have to be added (and also some conditions for the unique determination of the electric field in $\Omega_{I}$ ). The magnetic permeability $\boldsymbol{\mu}$ is assumed to be a symmetric tensor, uniformly positive definite in $\Omega$, with entries in $L^{\infty}(\Omega)$. The same assumption holds for the dielectric coefficient $\epsilon$ in $\Omega_{I}$ (this coefficient will come into play when imposing uniqueness conditions for $\mathbf{E}_{I}$ ), and for the electric conductivity $\sigma$ in $\Omega_{C}$; on the other hand, $\sigma$ is vanishing outside $\Omega_{C}$.

We will distinguish between two different geometric situations, and three different types of boundary conditions.

First geometric case: electric ports. The conductor $\Omega_{C}$ is not strictly contained in $\Omega$, namely, $\partial \Omega_{C} \cap \partial \Omega \neq \emptyset$. More precisely, for the sake of simplicity we assume that $\Omega_{C}$ is a simply connected domain with $\partial \Omega_{C} \cap \partial \Omega=\Gamma_{E} \cup \Gamma_{J}$, where $\Gamma_{E}$ and $\Gamma_{J}$ are connected and disjoint surfaces on $\partial \Omega$ ("electric ports"). Therefore, we have $\partial \Omega_{C}=\Gamma_{E} \cup \Gamma_{J} \cup \Gamma$. The boundary of the insulator $\Omega_{I}$, which is connected, is given by $\partial \Omega_{I}=\Gamma_{D} \cup \Gamma$, where $\Gamma_{D} \subset \partial \Omega$. As a consequence, we have $\partial \Gamma=\partial \Gamma_{D}=\partial \Gamma_{E} \cup \partial \Gamma_{J}$ (see Fig.1, left).

Second geometric case: internal conductor. The conductor $\Omega_{C}$ is strictly contained in $\Omega$, namely, $\partial \Omega_{C} \cap \partial \Omega=\emptyset$. Moreover, for the sake of 
simplicity we assume that $\Omega_{C}$ is a torus-like domain. In this case, we simply have $\partial \Omega_{C}=\Gamma$ and $\partial \Omega_{I}=\partial \Omega \cup \Gamma$ (see Fig.1, right).
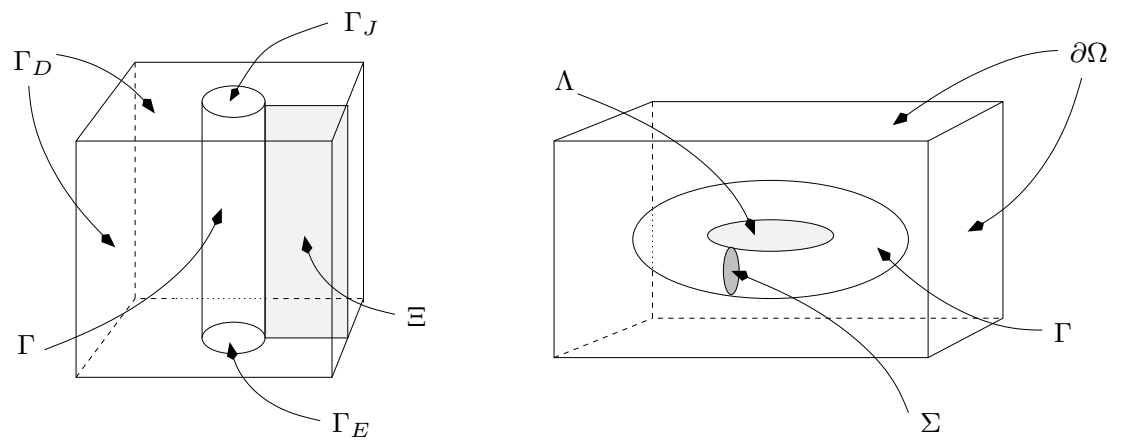

Figure 1: The geometry of the domain for the electric port case (left) and for the internal conductor case (right).

First set of boundary conditions. It is given by $\mathbf{E} \times \mathbf{n}=\mathbf{0}$ on $\partial \Omega$, for both the geometric cases.

Second set of boundary conditions. It is given by $\mathbf{E} \times \mathbf{n}=\mathbf{0}$ on $\Gamma_{E} \cup \Gamma_{J}$, and $\mathbf{H} \times \mathbf{n}=\mathbf{0}$ and $\boldsymbol{\epsilon} \mathbf{E} \cdot \mathbf{n}=0$ on $\Gamma_{D}$ for the electric port case, and by $\mathbf{H} \times \mathbf{n}=\mathbf{0}$ and $\boldsymbol{\epsilon} \mathbf{E} \cdot \mathbf{n}=0$ on $\partial \Omega$ for the internal conductor case.

Third set of boundary conditions. It is given by $\mathbf{E} \times \mathbf{n}=\mathbf{0}$ on $\Gamma_{E} \cup \Gamma_{J}$, $\boldsymbol{\mu} \mathbf{H} \cdot \mathbf{n}=0$ and $\boldsymbol{\epsilon} \mathbf{E} \cdot \mathbf{n}=0$ on $\Gamma_{D}$ for the electric port case, and by $\boldsymbol{\mu} \mathbf{H} \cdot \mathbf{n}=0$ and $\boldsymbol{\epsilon E} \cdot \mathbf{n}=0$ on $\partial \Omega$ for the internal conductor case.

Summing up, we are going to consider six different problems:

- Case A. Electric ports, $\mathbf{E} \times \mathbf{n}=\mathbf{0}$ on $\partial \Omega$

- Case B. Electric ports, $\mathbf{E} \times \mathbf{n}=\mathbf{0}$ on $\Gamma_{E} \cup \Gamma_{J}, \mathbf{H} \times \mathbf{n}=\mathbf{0}$ and $\boldsymbol{\epsilon} \mathbf{E} \cdot \mathbf{n}=0$ on $\Gamma_{D}$

- Case C. Electric ports, $\mathbf{E} \times \mathbf{n}=\mathbf{0}$ on $\Gamma_{E} \cup \Gamma_{J}, \boldsymbol{\mu H} \cdot \mathbf{n}=0$ and $\boldsymbol{\epsilon} \mathbf{E} \cdot \mathbf{n}=0$ on $\Gamma_{D}$

- Case D. Internal conductor, $\mathbf{E} \times \mathbf{n}=\mathbf{0}$ on $\partial \Omega$

- Case E. Internal conductor, $\mathbf{H} \times \mathbf{n}=\mathbf{0}$ and $\boldsymbol{\epsilon E} \cdot \mathbf{n}=0$ on $\partial \Omega$

- Case F. Internal conductor, $\boldsymbol{\mu} \mathbf{H} \cdot \mathbf{n}=0$ and $\boldsymbol{\epsilon} \mathbf{E} \cdot \mathbf{n}=0$ on $\partial \Omega$

Between the six boundary value problems described here above, Case $\mathrm{C}$ has some specific features. In fact, it is the only one for which the solution of the eddy-current problem is not unique.

Let us start by proving this result. 
Theorem 1.1. Let us consider the solutions $\mathbf{H}$ and $\mathbf{E}$ of the eddy-current problem

$$
\begin{array}{ll}
\operatorname{curl} \mathbf{H}-\boldsymbol{\sigma} \mathbf{E}=\mathbf{J}_{e} & \text { in } \Omega \\
\operatorname{curl} \mathbf{E}+i \omega \boldsymbol{\mu} \mathbf{H}=\mathbf{0} & \text { in } \Omega .
\end{array}
$$

The magnetic field $\mathbf{H}$ in $\Omega$ and the electric field $\mathbf{E}_{C}$ in $\Omega_{C}$ are uniquely determined for each one of the set of boundary conditions described in Cases A, B, $D, E$ and $F$.

Proof. Assume that $\mathbf{J}_{e}=\mathbf{0}$ in $\Omega$. Multiply the Faraday equation by $\overline{\mathbf{H}}$ and integrate in $\Omega$. Integration by parts gives

$0=\int_{\Omega} \operatorname{curl} \mathbf{E} \cdot \overline{\mathbf{H}}+\int_{\Omega} i \omega \boldsymbol{\mu} \mathbf{H} \cdot \overline{\mathbf{H}}=\int_{\Omega} \mathbf{E} \cdot \operatorname{curl} \overline{\mathbf{H}}+\int_{\Omega} i \omega \boldsymbol{\mu} \mathbf{H} \cdot \overline{\mathbf{H}}+\int_{\partial \Omega} \mathbf{n} \times \mathbf{E} \cdot \overline{\mathbf{H}}$.

Replacing $\mathbf{E}_{C}$ by $\boldsymbol{\sigma}^{-1} \operatorname{curl} \mathbf{H}_{C}$, and recalling that $\operatorname{curl} \mathbf{H}_{I}=\mathbf{0}$ in $\Omega_{I}$, we have

$$
0=\int_{\Omega_{C}} \boldsymbol{\sigma}^{-1} \operatorname{curl} \mathbf{H}_{C} \cdot \operatorname{curl} \overline{\mathbf{H}}_{C}+\int_{\Omega} i \omega \boldsymbol{\mu} \mathbf{H} \cdot \overline{\mathbf{H}}+\int_{\partial \Omega} \mathbf{n} \times \mathbf{E} \cdot \overline{\mathbf{H}} .
$$

Thus the uniqueness result follows at once if we prove that the boundary integral vanishes.

This is clearly the case if we are considering Cases A, B, D and E. For Case $\mathrm{F}$, we have $\operatorname{div}_{\tau}(\mathbf{E} \times \mathbf{n})=\operatorname{curl} \mathbf{E} \cdot \mathbf{n}=-i \omega \boldsymbol{\mu H} \cdot \mathbf{n}=0$ on $\partial \Omega$, hence there exists a scalar function $W$ such that $\mathbf{E} \times \mathbf{n}=\operatorname{grad} W \times \mathbf{n}$ on $\partial \Omega$. Therefore

$$
\begin{aligned}
\int_{\partial \Omega} \mathbf{n} \times \mathbf{E} \cdot \overline{\mathbf{H}} & =\int_{\partial \Omega} \overline{\mathbf{H}} \times \mathbf{n} \cdot \operatorname{grad} W=-\int_{\partial \Omega} \operatorname{div}_{\tau}(\overline{\mathbf{H}} \times \mathbf{n}) W \\
& =-\int_{\partial \Omega} \operatorname{curl} \overline{\mathbf{H}} \cdot \mathbf{n} W=0,
\end{aligned}
$$

as $\operatorname{curl} \mathbf{H}_{I}=\mathbf{0}$ in $\Omega_{I}$.

Remark 1.2. It is worthy to note that, for Case C, assuming $\mathbf{J}_{e}=\mathbf{0}$ one has

$$
\int_{\Omega_{C}} \boldsymbol{\sigma}^{-1} \operatorname{curl} \mathbf{H}_{C} \cdot \operatorname{curl} \overline{\mathbf{H}}_{C}+\int_{\Omega} i \omega \boldsymbol{\mu} \mathbf{H} \cdot \overline{\mathbf{H}}=W_{\mid \Gamma_{J}} \int_{\Gamma_{J}} \operatorname{curl} \overline{\mathbf{H}}_{C} \cdot \mathbf{n} .
$$

In fact, we know that on the ports $\Gamma_{E}$ and $\Gamma_{J}$ we still have $\operatorname{div}_{\tau}(\mathbf{E} \times \mathbf{n})=0$, and also on $\Gamma_{D}$ we have $\operatorname{div}_{\tau}(\mathbf{E} \times \mathbf{n})=\operatorname{curl} \mathbf{E} \cdot \mathbf{n}=-i \omega \boldsymbol{\mu} \mathbf{H} \cdot \mathbf{n}=0$, thus we conclude that $\operatorname{div}_{\tau}(\mathbf{E} \times \mathbf{n})=0$ on $\partial \Omega$. Therefore, as before we can write $\mathbf{E} \times \mathbf{n}=\operatorname{grad} W \times \mathbf{n}$ on $\partial \Omega$, and we see that $W$ is constant on $\Gamma_{E}$ and on $\Gamma_{J}$, say, $W=0$ on $\Gamma_{E}$ and $W=W_{J}$ on $\Gamma_{J}$. Thus

$$
\int_{\partial \Omega} \mathbf{n} \times \mathbf{E} \cdot \overline{\mathbf{H}}=-\int_{\partial \Omega} \operatorname{curl} \overline{\mathbf{H}} \cdot \mathbf{n} W=-W_{J} \int_{\Gamma_{J}} \operatorname{curl} \overline{\mathbf{H}}_{C} \cdot \mathbf{n},
$$

as $\operatorname{curl} \mathbf{H}_{I} \cdot \mathbf{n}=0$ on $\Gamma_{D}$ and $W=0$ on $\Gamma_{E}$.

In particular, we see that there is still a free degree of freedom: it can be either the constant value of $W$ on $\Gamma_{J}$ (the voltage between the two ports of 
$\Omega_{C}$ ), or the value $\int_{\Gamma_{J}} \operatorname{curl} \mathbf{H}_{C} \cdot \mathbf{n}$ (the current intensity in $\Omega_{C}$ ). Therefore, in the present case uniqueness requires that one of these conditions is also imposed.

Case $\mathrm{C}$ has been proposed in [14] as a valid approximation of a realistic electric port problem. Thus we start from it for our considerations.

In [5] (see also [8]) it has been proved that

Theorem 1.3. For each $\mathbf{J}_{e} \in\left(L^{2}(\Omega)\right)^{3}$, there exists a unique solution $\mathbf{H}$ and $\mathbf{E}$ of the eddy-current problem (Case $C$ )

$$
\begin{array}{ll}
\operatorname{curl} \mathbf{H}-\boldsymbol{\sigma} \mathbf{E}=\mathbf{J}_{e} & \text { in } \Omega \\
\operatorname{curl} \mathbf{E}+i \omega \boldsymbol{\mu} \mathbf{H}=\mathbf{0} & \text { in } \Omega \\
\operatorname{div}\left(\boldsymbol{\epsilon}_{I} \mathbf{E}_{I}\right)=0 & \text { in } \Omega_{I} \\
\mathbf{E} \times \mathbf{n}=\mathbf{0} & \text { on } \Gamma_{E} \cup \Gamma_{J} \\
\boldsymbol{\epsilon} \mathbf{E} \cdot \mathbf{n}=0 & \text { on } \Gamma_{D} \\
\boldsymbol{\mu} \mathbf{H} \cdot \mathbf{n}=0 & \text { on } \Gamma_{D},
\end{array}
$$

with one of the following additional conditions:

$$
\text { either } \quad W_{J}=V \quad \text { or } \quad \int_{\Gamma_{J}} \operatorname{curl} \mathbf{H}_{C} \cdot \mathbf{n}=I,
$$

where the voltage $V$ and the current intensity $I$ are given complex numbers, and $W_{J}$ denotes the constant value on $\Gamma_{J}$ of the function $W$ such that $\mathbf{E} \times \mathbf{n}=$ $\operatorname{grad} W \times \mathbf{n}$ on $\partial \Omega$, having set $W=0$ on $\Gamma_{E}$.

In [8] and [5] the convergence of a finite element approximation scheme is also proved (in the former the considered unknowns are $\mathbf{H}_{C}$ and a scalar magnetic potential, in the latter the same magnetic potential and $\mathbf{E}_{C}$ ).

Other finite element schemes can be found in [23] and [10], where the problem is described through the so-called T- $\Phi$ formulation, namely, in terms of a current vector potential and a scalar magnetic potential.

\section{The power law}

Hiptmair and Sterz in [20], a paper that has deeply inspired our work, have proposed to use a suitable power law to relate the voltage and the current intensity. They define

$$
\widehat{P}:=\int_{\Omega_{C}} \boldsymbol{\sigma} \mathbf{E}_{C} \cdot \overline{\mathbf{E}}_{C}+i \omega \int_{\Omega} \boldsymbol{\mu} \mathbf{H} \cdot \overline{\mathbf{H}}
$$

and assume that, for the problem at hand, one has $\widehat{P}=V \bar{I}$.

In this paper we propose to modify the definition of the power in the following way:

$$
P:=\int_{\Omega_{C}} \boldsymbol{\sigma}^{-1} \operatorname{curl} \mathbf{H}_{C} \cdot \operatorname{curl} \overline{\mathbf{H}}_{C}+i \omega \int_{\Omega} \boldsymbol{\mu} \mathbf{H} \cdot \overline{\mathbf{H}}
$$


Since curl $\mathbf{H}=\boldsymbol{\sigma} \mathbf{E}+\mathbf{J}_{e}$, when $\mathbf{J}_{e, C}=\mathbf{0}$ the two definitions are clearly the same: but we will see in the following that the presence of the current density has important consequences.

To motivate the choice of our power law, let us consider the eddy-current problem (1) (Case C) with a given assigned voltage $V$ and with $\mathbf{J}_{e, I}=\mathbf{0}$, $\mathbf{J}_{e, C}=-V \boldsymbol{\sigma} \operatorname{grad} \phi_{C}$, where $\phi_{C}$ is the unique solution to

$$
\begin{cases}\operatorname{div}\left(\boldsymbol{\sigma} \operatorname{grad} \phi_{C}\right)=0 & \text { in } \Omega_{C} \\ \phi_{C}=1 & \text { on } \Gamma_{J} \\ \phi_{C}=0 & \text { on } \Gamma_{E} \\ \boldsymbol{\sigma} \operatorname{grad} \phi_{C} \cdot \mathbf{n}=0 & \text { on } \Gamma .\end{cases}
$$

It is easily seen that the solution is given by $\mathbf{E}=V \operatorname{grad} \phi$ and $\mathbf{H}=\mathbf{0}$, where $\phi$ is equal to $\phi_{C}$ in $\Omega_{C}$ and to $\phi_{I}$ in $\Omega_{I}, \phi_{I}$ being the unique solution to

$$
\begin{cases}\operatorname{div}\left(\boldsymbol{\epsilon}_{I} \operatorname{grad} \phi_{I}\right)=0 & \text { in } \Omega_{I} \\ \phi_{I}=\phi_{C} & \text { on } \Gamma \\ \boldsymbol{\epsilon}_{I} \operatorname{grad} \phi_{I} \cdot \mathbf{n}=0 & \text { on } \Gamma_{D} .\end{cases}
$$

Therefore the current intensity $I$ is equal to 0 , and the power law $P=V \bar{I}$ is clearly satisfied, whereas one has $\widehat{P} \neq 0=V \bar{I}$.

The power law $P=V \bar{I}$ is also giving us some other useful informations. In fact, for each complex number $q \in \mathbb{C}$ take now $\mathbf{J}_{e, C}=q \boldsymbol{\sigma} \operatorname{grad} \phi_{C}, \mathbf{J}_{e, I}=\mathbf{0}$. Computing the power for the corresponding solution we find, by proceeding as in Theorem 1.1 and in Remark 1.2,

$$
\begin{aligned}
P & =\int_{\Omega_{C}} \boldsymbol{\sigma}^{-1} \operatorname{curl} \mathbf{H}_{C} \cdot \operatorname{curl} \overline{\mathbf{H}}_{C}+i \omega \int_{\Omega} \boldsymbol{\mu} \mathbf{H} \cdot \overline{\mathbf{H}} \\
& =\int_{\Omega_{C}} \boldsymbol{\sigma}^{-1} \mathbf{J}_{e, C} \cdot \operatorname{curl} \overline{\mathbf{H}}_{C}+V \int_{\Gamma_{J}} \operatorname{curl} \overline{\mathbf{H}}_{C} \cdot \mathbf{n} \\
& =q \int_{\Omega_{C}} \operatorname{grad} \phi_{C} \cdot \operatorname{curl} \overline{\mathbf{H}}_{C}+V \int_{\Gamma_{J}} \operatorname{curl} \overline{\mathbf{H}}_{C} \cdot \mathbf{n} .
\end{aligned}
$$

On the other hand,

$$
\begin{aligned}
\int_{\Omega_{C}} \operatorname{grad} & \phi_{C} \cdot \operatorname{curl} \overline{\mathbf{H}}_{C} \\
& =-\int_{\Omega_{C}} \phi_{C} \operatorname{div} \operatorname{curl} \overline{\mathbf{H}}_{C}+\int_{\Gamma_{E} \cup \Gamma_{J} \cup \Gamma} \phi_{C} \operatorname{curl} \overline{\mathbf{H}}_{C} \cdot \mathbf{n}_{C} \\
& =\int_{\Gamma_{J}} \operatorname{curl} \overline{\mathbf{H}}_{C} \cdot \mathbf{n} .
\end{aligned}
$$

as $\phi_{C}=0$ on $\Gamma_{E}, \phi_{C}=1$ on $\Gamma_{J}$ and $\operatorname{curl} \mathbf{H}_{C} \cdot \mathbf{n}_{C}=\operatorname{curl} \mathbf{H}_{I} \cdot \mathbf{n}_{C}=0$ on $\Gamma$.

In conclusion,

$$
P=(q+V) \int_{\Gamma_{J}} \operatorname{curl} \overline{\mathbf{H}}_{C} \cdot \mathbf{n}=(q+V) \bar{I} .
$$

This is telling us that, when considering Case C, assigning a voltage $V$ is in some sense equivalent to impose a current density $\mathbf{J}_{e, C}=V \boldsymbol{\sigma} \operatorname{grad} \phi_{C}$ in $\Omega_{C}$.

More precisely, the solution $(\widetilde{\mathbf{H}}, \widetilde{\mathbf{E}})$ with voltage $V$ and $\mathbf{J}_{e, C}=\mathbf{0}$ and the solution $(\widehat{\mathbf{H}}, \widehat{\mathbf{E}})$ with voltage 0 and $\mathbf{J}_{e, C}=V \operatorname{grad} \phi_{C}$ satisfy $\widetilde{\mathbf{H}}=\widehat{\mathbf{H}}$ : in fact, the difference $(\widetilde{\mathbf{H}}-\widehat{\mathbf{H}}, \widetilde{\mathbf{E}}-\widehat{\mathbf{E}})$ is a solution of the problem with voltage $V$ and 
$\mathbf{J}_{e, C}=-V \operatorname{grad} \phi_{C}$, therefore, as we have seen above, $\widetilde{\mathbf{E}}-\widehat{\mathbf{E}}=V \operatorname{grad} \phi$ and $\widetilde{\mathbf{H}}-\widehat{\mathbf{H}}=\mathbf{0}$.

This will lead us to propose a suitable formulation for the eddy-current problem with one of the boundary conditions described in Cases A, B, D, E, $\mathrm{F}$, and moreover subjected to a given voltage or current intensity excitation: the key point will be that these excitations, differently from Case C, have to be interpreted as a particular applied current density.

Remark 2.1. Clearly, the power law $P=V \bar{I}$ does not hold if $\mathbf{J}_{e, I} \neq \mathbf{0}$ or $\mathbf{J}_{e, C} \neq \mathbf{0}$, and in these cases the injected power has a more general expression than $V \bar{I}$, namely, it is easily checked that

$$
\begin{aligned}
P & =\int_{\Omega_{C}} \boldsymbol{\sigma}^{-1} \operatorname{curl} \mathbf{H}_{C} \cdot \operatorname{curl} \overline{\mathbf{H}}_{C}+i \omega \int_{\Omega} \boldsymbol{\mu} \mathbf{H} \cdot \overline{\mathbf{H}} \\
& =\int_{\Omega_{C}} \boldsymbol{\sigma}^{-1} \mathbf{J}_{e, C} \cdot \operatorname{curl} \overline{\mathbf{H}}_{C}-\int_{\Omega_{I}} \overline{\mathbf{J}}_{e, I} \cdot \mathbf{E}_{I}+V \bar{I}
\end{aligned}
$$

On the other hand, when $\mathbf{J}_{e, I}=\mathbf{0}$ and $\mathbf{J}_{e, C}=q \boldsymbol{\sigma} \operatorname{grad} \phi_{C}$, we have seen that for Case $\mathrm{C}$ the power law still holds, in the generalized form $P=(V+q) \bar{I}$. This is showing that we have to consider two voltages, say, an "electric" voltage $V$ (the value $V=W_{\mid \Gamma_{J}}-W_{\mid \Gamma_{E}}$, where the electric field satisfies $\mathbf{E} \times \mathbf{n}=\operatorname{grad} W \times \mathbf{n}$ on $\partial \Omega$ ), and a "source" voltage $q$, associated to the current density $q \boldsymbol{\sigma} \operatorname{grad} \phi_{C}$ : their sum $V+q$ is the total voltage.

When considering the other cases A, B, D, E and F, only the "source" voltage keeps a meaning.

\section{Voltage and current excitation}

As the eddy-current problem has a unique solution for each one of the set of boundary conditions described in Cases A, B, D, E and F (see Theorem 1.1), it is not possible to impose an additional condition, say, voltage or current intensity, if we do not relax some of the imposed equations.

Before starting, let us mention the formulations proposed in some preceding papers. In [20], where the voltage/current excitation problem has been considered in the most systematic way, it was proposed to modify a little the formulation for Case A, requiring $\mathbf{E} \times \mathbf{n}=\operatorname{grad} \varphi \times \mathbf{n}$ on $\partial \Omega$, where $\varphi \in H^{1 / 2}(\partial \Omega)$, $\varphi=V$ on $\Gamma_{J}, \varphi=0$ on $\Gamma_{E}$ (and, therefore, $\varphi \neq$ const. in a transition region $\left.\Theta \subset \Gamma_{D}\right)$. In other words, $\mathbf{E} \times \mathbf{n} \neq \mathbf{0}$ in $\Theta$. This formulation, which is proved to be well-posed, depends however on the choice of the region $\Theta$ and of the function $\varphi$ in $\Theta$. An alternative approach, also proposed in [20], valid for all the cases here considered and for which $\Theta=\emptyset$, ends up with the violation of the Faraday law on a specific surface (either the surface that "cuts" the basic non-bounding cycle in $\Omega_{I}$, or else any surface crossing the interface $\Gamma$ ).

In [17] and [25] the internal conductor case is considered, having assigned a given voltage $V$. Also in this case the Faraday law is violated on the cutting surface $\Lambda$. Instead, the approach proposed in [18] gives a solution that does not satisfy the Faraday law across the interface $\Gamma$. 
In [8] a formulation for the electric port case with assigned current intensity is given, leading to the solution also obtained in [5] for Case $\mathrm{C}$; however, for Case A it can be checked that the Faraday law is violated on the cutting surface $\Xi$ (instead, the violation of the Faraday law across the interface $\Gamma$ occurs in [6], where the internal conductor case is considered).

Finally, in [23] and [10] the finite element approximation of Case C is considered for an assigned voltage, by means of a formulation based on a current vector potential and a magnetic scalar potential.

Let us come now to our point of view: clearly, on one side we do not want to renounce to Maxwell equations, namely, to Faraday and Ampère equations; on the other side, we would like to formulate a problem for which only the physical quantities and the physical domains $\Omega_{C}$ and $\Omega_{I}$ play a role (and not artificial regions like, e.g., the transition zone $\Theta$ introduced in [20]).

The main point is to recall what we have proved for Case $\mathrm{C}$, where a voltage $V$ was "equivalent" (at least, for the determination of $\mathbf{H}$ and in the power law) to the current density $\mathbf{J}_{e, C}=V \boldsymbol{\sigma} \operatorname{grad} \phi_{C}$ in $\Omega_{C}$. Note that the function grad $\phi_{C}$ is the basis function of the space of harmonic fields

$$
\begin{array}{r}
\widehat{\mathcal{H}}\left(\Omega_{C}\right):=\left\{\widehat{\boldsymbol{\eta}} \in\left(L^{2}\left(\Omega_{C}\right)\right)^{3} \mid \operatorname{curl} \widehat{\boldsymbol{\eta}}=\mathbf{0}, \operatorname{div}(\boldsymbol{\sigma} \widehat{\boldsymbol{\eta}})=0,\right. \\
\left.\boldsymbol{\sigma} \widehat{\boldsymbol{\eta}} \cdot \mathbf{n}=0 \text { on } \Gamma, \widehat{\boldsymbol{\eta}} \times \mathbf{n}=\mathbf{0} \text { on } \Gamma_{E} \cup \Gamma_{J}\right\},
\end{array}
$$

normalized with the condition

$$
\int_{\widehat{\gamma}} \widehat{\boldsymbol{\eta}} \cdot d \boldsymbol{\tau}=1,
$$

the path $\widehat{\gamma}$ joining $\Gamma_{E}$ to $\Gamma_{J}$. Thus, for the internal conductor case we are led to introduce the space of harmonic fields

$$
\mathcal{H}\left(\Omega_{C}\right):=\left\{\boldsymbol{\eta} \in\left(L^{2}\left(\Omega_{C}\right)\right)^{3} \mid \operatorname{curl} \boldsymbol{\eta}=\mathbf{0}, \operatorname{div}(\boldsymbol{\sigma} \boldsymbol{\eta})=0, \boldsymbol{\sigma} \boldsymbol{\eta} \cdot \mathbf{n}=0 \text { on } \Gamma\right\},
$$

defining as $\boldsymbol{\rho}_{C}$ its basis function normalized with the condition

$$
\int_{\gamma} \boldsymbol{\rho}_{C} \cdot d \boldsymbol{\tau}=1,
$$

where the (closed) cycle $\gamma$ is internal to $\Omega_{C}$ (and we have freely chosen an orientation of $\gamma)$.

The voltage or current excitation problem is therefore formulated as follows.

Voltage rule. When a voltage $V$ is imposed, modify the Ohm law in $\Omega_{C}$ adding to the current density $\boldsymbol{\sigma} \mathbf{E}_{C}$ the "applied" current density $\mathbf{J}_{e, C}=V \boldsymbol{\sigma} \mathbf{Q}_{C}$, where $\mathbf{Q}_{C}=\operatorname{grad} \phi_{C}$ for the electric port case, and $\mathbf{Q}_{C}=\boldsymbol{\rho}_{C}$ for the internal conductor case. Thus the Ampère law reads

$$
\operatorname{curl} \mathbf{H}_{C}=\boldsymbol{\sigma} \mathbf{E}_{C}+V \boldsymbol{\sigma} \mathbf{Q}_{C} .
$$

In the former case, we intend that the voltage passes from 0 on $\Gamma_{E}$ to $V$ on $\Gamma_{J}$; in the latter case, the voltage passes from 0 to $V$ along the basic cycle $\gamma$. 
Current intensity rule. When a current itensity $I$ is imposed, determine the voltage $V$ and modify the Ohm law in $\Omega_{C}$ adding to the current density $\boldsymbol{\sigma} \mathbf{E}_{C}$ the "applied" current density $\mathbf{J}_{e, C}=V \boldsymbol{\sigma} \mathbf{Q}_{C}$, where $\mathbf{Q}_{C}$ is as in the "voltage rule". Thus the Ampère law reads

$$
\operatorname{curl} \mathbf{H}_{C}=\sigma \mathbf{E}_{C}+V \boldsymbol{\sigma} \mathbf{Q}_{C},
$$

and moreover the constraint

$$
\int_{S} \operatorname{curl} \mathbf{H}_{C} \cdot \mathbf{n}=I
$$

has to be satisfied, where $S=\Gamma_{J}$ for the electric port case, and $S=\Sigma$, a section of $\Omega_{C}$, for the internal conductor case. In the former case, the unit vector $\mathbf{n}$ is the outward normal on $\Gamma_{J}$; in the latter case, the unit vector $\mathbf{n}$ on $\Sigma$ is oriented as the basic cycle $\gamma$.

Let us show that, when adopting these two rules, we are respecting the power law. Assume that we have $\mathbf{J}_{e, C}=V \boldsymbol{\sigma} \mathbf{Q}_{C}$ and $\mathbf{J}_{e, I}=\mathbf{0}$. Then, by proceeding as in Theorem 1.1, and taking into account the boundary conditions of Cases A, B, D, E and F, we have that

$$
\int_{\Omega_{C}} \boldsymbol{\sigma}^{-1} \operatorname{curl} \mathbf{H}_{C} \cdot \operatorname{curl} \overline{\mathbf{H}}_{C}+i \omega \int_{\Omega} \boldsymbol{\mu} \mathbf{H} \cdot \overline{\mathbf{H}}=\int_{\Omega_{C}} \boldsymbol{\sigma}^{-1} \mathbf{J}_{e, C} \cdot \operatorname{curl} \overline{\mathbf{H}}_{C},
$$

hence

$$
P=\int_{\Omega_{C}} \boldsymbol{\sigma}^{-1} \operatorname{curl} \mathbf{H}_{C} \cdot \operatorname{curl} \overline{\mathbf{H}}_{C}+i \omega \int_{\Omega} \boldsymbol{\mu} \mathbf{H} \cdot \overline{\mathbf{H}}=V \int_{\Omega_{C}} \mathbf{Q}_{C} \cdot \operatorname{curl} \overline{\mathbf{H}}_{C} .
$$

On the other hand, from (7) we have

$$
\int_{\Omega_{C}} \operatorname{grad} \phi_{C} \cdot \operatorname{curl} \overline{\mathbf{H}}_{C}=\int_{\Gamma_{J}} \operatorname{curl} \overline{\mathbf{H}}_{C} \cdot \mathbf{n}=\bar{I},
$$

thus if $\mathbf{Q}_{C}=\operatorname{grad} \phi_{C}$ we conclude with

$$
\begin{aligned}
P & =\int_{\Omega_{C}} \boldsymbol{\sigma}^{-1} \operatorname{curl} \mathbf{H}_{C} \cdot \operatorname{curl} \overline{\mathbf{H}}_{C}+i \omega \int_{\Omega} \boldsymbol{\mu} \mathbf{H} \cdot \overline{\mathbf{H}} \\
& =V \int_{\Omega_{C}} \operatorname{grad} \phi_{C} \cdot \operatorname{curl} \overline{\mathbf{H}}_{C}=V \bar{I},
\end{aligned}
$$

the power law for the electric port case.

The internal conductor case needs some additional informations, in order to express the current intensity in a suitable way. Let us denote by $\Sigma$ a section of $\Omega_{C}$, namely, a surface in $\Omega_{C}$ cutting the basic non-bounding cycle $\gamma$. We know that the basis function $\boldsymbol{\rho}_{C}$ is the $L^{2}\left(\Omega_{C}\right)$-extension of the gradient of a suitable scalar function $q$, defined in $\Omega_{C} \backslash \Sigma$ and having a jump equal to 1 across $\Sigma$. Hence,

$$
\begin{aligned}
\int_{\Omega_{C}} \operatorname{curl} \mathbf{H}_{C} \cdot \boldsymbol{\rho}_{C} & =\int_{\Omega_{C} \backslash \Sigma} \operatorname{curl} \mathbf{H}_{C} \cdot \operatorname{grad} q \\
& =-\int_{\Omega_{C} \backslash \Sigma} q \operatorname{div} \operatorname{curl} \mathbf{H}_{C}+\int_{\Gamma} q \operatorname{curl} \mathbf{H}_{C} \cdot \mathbf{n}_{C} \\
& \quad+\int_{\Sigma} \operatorname{curl} \mathbf{H}_{C} \cdot \mathbf{n} \\
& =\int_{\Sigma} \operatorname{curl} \mathbf{H}_{C} \cdot \mathbf{n},
\end{aligned}
$$


as curl $\mathbf{H}_{C} \cdot \mathbf{n}_{C}=\operatorname{curl} \mathbf{H}_{I} \cdot \mathbf{n}_{C}=0$ on $\Gamma$ and the jump of $q$ on $\Sigma$ is equal to 1 . Hence we end up with

$$
\begin{aligned}
P & =\int_{\Omega_{C}} \boldsymbol{\sigma}^{-1} \operatorname{curl} \mathbf{H}_{C} \cdot \operatorname{curl} \overline{\mathbf{H}}_{C}+i \omega \int_{\Omega} \boldsymbol{\mu} \mathbf{H} \cdot \overline{\mathbf{H}} \\
& =V \int_{\Omega_{C}} \boldsymbol{\rho}_{C} \cdot \operatorname{curl} \overline{\mathbf{H}}_{C}=V \bar{I},
\end{aligned}
$$

the power law for the internal conductor case.

Remark 3.1. As it is clear from our procedure, in the electric port case we could obtain a suitable formulation (namely, satisfying the power law) for any current density $\mathbf{J}_{e, C}=V \boldsymbol{\sigma} \operatorname{grad} \Phi_{C}$ such that $\Phi_{C}=1$ on $\Gamma_{J}$ and $\Phi_{C}=0$ on $\Gamma_{E}$. Hence, how to motivate the choice of $\phi_{C}$ introduced in (5)?

In this respect, it should be noted that, from the Ampère equation curl $\mathbf{H}_{C}=$ $\sigma \mathbf{E}_{C}+\mathbf{J}_{e, C}$, the electric field satisfies the (physically consistent) conditions $\operatorname{div}\left(\boldsymbol{\sigma} \mathbf{E}_{C}\right)=0$ in $\Omega_{C}$ and $\boldsymbol{\sigma} \mathbf{E}_{C} \cdot \mathbf{n}=0$ on $\Gamma$ only if $\operatorname{div} \mathbf{J}_{e, C}=0$ in $\Omega_{C}$ and $\mathbf{J}_{e, C} \cdot \mathbf{n}=0$ on $\Gamma$, therefore, only if $\Phi_{C}=\phi_{C}$, the solution to (5).

The same remark applies for the internal conductor case: in that situation, the integral

$$
\int_{\Omega_{C}} \mathbf{u}_{C} \cdot \operatorname{curl} \overline{\mathbf{H}}_{C}
$$

has the same value for any vector field $\mathbf{u}_{C}$ such that curl $\mathbf{u}_{C}=\mathbf{0}$ and $\int_{\gamma} \mathbf{u}_{C} \cdot d \boldsymbol{\tau}=$ 1. But if we also require that $\operatorname{div}\left(\boldsymbol{\sigma} \mathbf{u}_{C}\right)=0$ in $\Omega_{C}$ and $\boldsymbol{\sigma} \mathbf{u}_{C} \cdot \mathbf{n}=0$ on $\Gamma$, then we conclude $\mathbf{u}_{C}=\boldsymbol{\rho}_{C}$.

\section{Variational formulations}

We can consider $\mathbf{H}$-based formulations, or $\mathbf{E}$-based formulations. In our opinion, the simplest approach is in terms of $\mathbf{H}$. We will focus first on the electric port case; however, we do not present here Case C, that, for a "hybrid" formulation which is related to the $\mathbf{H}$-formulation, has been studied in [5]. Then we will consider the internal conductor case, whose formulation is quite similar, focusing in particular on Case F.

- Electric ports: voltage excitation, $\mathbf{H}$ formulation

For Case A, the problem is as follows: for each given $V \in \mathbb{C}$ find the unique solution $\mathbf{H} \in X$ to

$$
\int_{\Omega_{C}} \boldsymbol{\sigma}^{-1} \operatorname{curl} \mathbf{H}_{C} \cdot \operatorname{curl} \overline{\mathbf{w}}_{C}+\int_{\Omega} i \omega \boldsymbol{\mu} \mathbf{H} \cdot \overline{\mathbf{w}}=V \int_{\Omega_{C}} \operatorname{grad} \phi_{C} \cdot \operatorname{curl} \overline{\mathbf{w}}_{C}
$$

for each $\mathbf{w} \in X$, where

$$
X:=\left\{\mathbf{w} \in H(\operatorname{curl} ; \Omega) \mid \operatorname{curl} \mathbf{w}_{I}=\mathbf{0} \text { in } \Omega_{I}\right\} .
$$

Then set $\mathbf{E}_{C}:=\boldsymbol{\sigma}^{-1} \operatorname{curl} \mathbf{H}_{C}-V \operatorname{grad} \phi_{C}$ in $\Omega_{C}$, and in $\Omega_{I}$ define $\mathbf{E}_{I}$ to be the solution to

$$
\begin{cases}\operatorname{curl} \mathbf{E}_{I}=-i \omega \boldsymbol{\mu}_{I} \mathbf{H}_{I} & \text { in } \Omega_{I} \\ \operatorname{div}\left(\boldsymbol{\epsilon}_{I} \mathbf{E}_{I}\right)=0 & \text { in } \Omega_{I} \\ \mathbf{E}_{I} \times \mathbf{n}_{I}=-\mathbf{E}_{C} \times \mathbf{n}_{C} & \text { on } \Gamma \\ \mathbf{E}_{I} \times \mathbf{n}=\mathbf{0} & \text { on } \Gamma_{D} .\end{cases}
$$


Let us remark that the voltage excitation problem for Case B is trivial: in fact, from (7) and the Stokes theorem we have

$$
\int_{\Omega_{C}} \operatorname{grad} \phi_{C} \cdot \operatorname{curl} \overline{\mathbf{w}}_{C}=\int_{\Gamma_{J}} \operatorname{curl} \overline{\mathbf{w}}_{C} \cdot \mathbf{n}=\int_{\partial \Gamma_{J}} \overline{\mathbf{w}} \cdot d \boldsymbol{\tau}=0,
$$

as $\mathbf{w} \times \mathbf{n}=\mathbf{0}$ on $\Gamma_{D}$. Therefore, for any $V \in \mathbb{C}$ we find $\mathbf{H}=\mathbf{0}$; hence, we can assume $V=0$ and set $\mathbf{E}=\mathbf{0}$.

Well-posedness of problem (9) comes from the coerciveness in $X$ of the sesquilinear form $\int_{\Omega_{C}} \boldsymbol{\sigma}^{-1} \operatorname{curl} \mathbf{H}_{C} \cdot \operatorname{curl} \overline{\mathbf{w}}_{C}+\int_{\Omega} i \omega \boldsymbol{\mu H} \cdot \overline{\mathbf{w}}$.

Instead, a delicate point here is the unique solvability of Problem (10). In fact, as it is well-known, boundary-value problems for the curl-div system in general need that some compatibility conditions are satisfied in order to assure the existence of a solution, and that suitable additional conditions are imposed to guarantee its uniqueness; some of these conditions are related to the nontrivial topology of $\Omega_{I}$.

More precisely, first one has to verify the conditions $\operatorname{div}\left(\boldsymbol{\mu}_{I} \mathbf{H}_{I}\right)=0$ in $\Omega_{I}$, $\operatorname{div}_{\tau}\left(\mathbf{E}_{C} \times \mathbf{n}_{C}\right)=i \omega \boldsymbol{\mu}_{I} \mathbf{H}_{I} \cdot \mathbf{n}_{I}$ on $\Gamma$, and $\boldsymbol{\mu}_{I} \mathbf{H}_{I} \cdot \mathbf{n}_{I}=0$ on $\Gamma_{D}$.

It is possible to check that these conditions are satisfied by means of a suitable choice of test functions in (9) (for a similar procedure, see, for instance, $[2])$. In fact, the first follows from (9) taking as test function $\mathbf{w}=\operatorname{grad} \psi, \psi$ a smooth function with a compact support in $\Omega$ (and in this way one also obtains $\boldsymbol{\mu}_{C} \mathbf{H}_{C} \cdot \mathbf{n}_{C}+\boldsymbol{\mu}_{I} \mathbf{H}_{I} \cdot \mathbf{n}_{I}=0$ on $\Gamma$, as, indeed, $\operatorname{div}(\boldsymbol{\mu} \mathbf{H})=0$ in $\Omega$ ). The second comes from the Faraday equation in $\Omega_{C}$, which is obtained by integration by parts, and the relation $\operatorname{div}_{\tau}\left(\mathbf{E}_{C} \times \mathbf{n}_{C}\right)=\operatorname{curl} \mathbf{E}_{C} \cdot \mathbf{n}_{C}$. The last is obtained by taking as test function $\mathbf{w}=\operatorname{grad} \psi$, with $\psi \in H^{1}(\Omega)$.

Then one has to consider some spaces of harmonic fields. Concerning uniqueness, it is clear that we are interested in requiring that the solution $\mathbf{E}_{I}$ is orthogonal (with weight $\boldsymbol{\epsilon}_{I}$ ) to the space

$$
\begin{aligned}
& \mathcal{H}_{A}^{\text {un }}\left(\Omega_{I}\right):=\left\{\widehat{\boldsymbol{\eta}} \in\left(L^{2}\left(\Omega_{I}\right)\right)^{3} \mid \operatorname{curl} \widehat{\boldsymbol{\eta}}=\mathbf{0}, \operatorname{div}\left(\boldsymbol{\epsilon}_{I} \widehat{\boldsymbol{\eta}}\right)=0,\right. \\
& \left.\widehat{\boldsymbol{\eta}} \times \mathbf{n}=\mathbf{0} \text { on } \Gamma_{D} \cup \Gamma\right\} \text {. }
\end{aligned}
$$

However, this space is trivial (namely, it contains only $\widehat{\boldsymbol{\eta}}=\mathbf{0}$ ). In fact, cutting $\Omega_{I}$ with a surface $\Xi$ transversal to $\Gamma_{D}$ and $\Gamma$, an element $\widehat{\boldsymbol{\eta}}$ of $\mathcal{H}_{A}^{\mathrm{un}}\left(\Omega_{I}\right)$ in the set $\Omega_{I} \backslash \Xi$ is the gradient of a function $p$ having a constant jump through $\Xi$. But, due to the fact that $\operatorname{grad} p \times \mathbf{n}=\mathbf{0}$ on $\Gamma_{D} \cup \Gamma$, a connected surface, $p$ is constant on $\Gamma_{D} \cup \Gamma$, and therefore its jump through $\Xi$ is equal to 0 . Thus $\widehat{\boldsymbol{\eta}}$ is the gradient of a harmonic function $p$ with constant boundary value: hence $p$ is constant in $\Omega_{I}$ and $\widehat{\boldsymbol{\eta}}=\mathbf{0}$ in $\Omega_{I}$.

Existence is instead associated to the space

$$
\begin{array}{r}
\mathcal{H}_{A}^{\mathrm{ex}}\left(\Omega_{I}\right):=\left\{\widehat{\boldsymbol{\eta}} \in\left(L^{2}\left(\Omega_{I}\right)\right)^{3} \mid \operatorname{curl} \widehat{\boldsymbol{\eta}}=\mathbf{0}, \operatorname{div} \widehat{\boldsymbol{\eta}}=0,\right. \\
\left.\widehat{\boldsymbol{\eta}} \cdot \mathbf{n}=0 \text { on } \Gamma_{D} \cup \Gamma\right\},
\end{array}
$$

which, proceeding as before, is easily showed to be one-dimensional; let us denote by $\widehat{\boldsymbol{\rho}}_{I}$ its basis vector. For the solvability of Problem (10) one has to satisfy the 
compatibility condition

$$
\int_{\Omega_{I}} i \omega \boldsymbol{\mu}_{I} \mathbf{H}_{I} \cdot \widehat{\boldsymbol{\rho}}_{I}+\int_{\Gamma} \mathbf{E}_{C} \times \mathbf{n}_{C} \cdot \widehat{\boldsymbol{\rho}}_{I}=0,
$$

which comes from $(10)_{1}$ and $(10)_{3}$ by integration by parts. This relation indeed follows from (9) by choosing the test function $\mathbf{w}_{I}=\widehat{\boldsymbol{\rho}}_{I}$ and $\mathbf{w}_{C}=\widehat{\boldsymbol{\rho}}^{*}$, where $\widehat{\boldsymbol{\rho}}^{*} \in H\left(\operatorname{curl} ; \Omega_{C}\right)$ satisfies $\widehat{\boldsymbol{\rho}}^{*} \times \mathbf{n}_{I}=\widehat{\boldsymbol{\rho}}_{I} \times \mathbf{n}_{I}$ on $\Gamma$, integrating by parts and using the Faraday equation in $\Omega_{C}$.

In conclusion, (10) is uniquely solvable. It is important to remark that this is not the case if one defines, as in [20], where the same formulation (9) has been proposed, the electric field $\mathbf{E}_{C}=\boldsymbol{\sigma}^{-1} \operatorname{curl} \mathbf{H}_{C}$ in $\Omega_{C}$ : in that case, in fact, (11) is not satisfied, and therefore it is not possible to determine $\mathbf{E}_{I}$.

It is worthy to note that (11) is indeed equivalent to the Faraday equation on the surface $\Xi$ that cuts the basic non-bounding cycle in $\Omega_{I}$. Hence, setting $\mathbf{E}_{C}=\sigma^{-1}$ curl $\mathbf{H}_{C}$ leads to the violation of the Faraday equation on that surface.

\section{- Electric ports: current intensity excitation, $\mathbf{H}$ formulation}

Let us start noting that this problem has not a meaning for Case B. In fact, one has

$$
I=\int_{\Gamma_{J}} \operatorname{curl} \mathbf{H}_{C} \cdot \mathbf{n}=\int_{\partial \Gamma_{J}} \mathbf{H} \cdot d \boldsymbol{\tau}=0,
$$

as $\mathbf{H} \times \mathbf{n}=\mathbf{0}$ on $\Gamma_{D}$.

Therefore, we only consider Case A. The problem can be expressed in this way: for each given $I \in \mathbb{C}$ find the unique solution $(\mathbf{H}, V) \in X \times \mathbb{C}$ to

$$
\begin{cases}\int_{\Omega_{C}} \boldsymbol{\sigma}^{-1} \operatorname{curl} \mathbf{H}_{C} \cdot \operatorname{curl} \overline{\mathbf{w}}_{C} & +\int_{\Omega} i \omega \boldsymbol{\mu} \mathbf{H} \cdot \overline{\mathbf{w}} \\ \int_{\Omega_{C}} \operatorname{grad} \phi_{C} \cdot \operatorname{curl} \mathbf{H}_{C}=I & -V \int_{\Omega_{C}} \operatorname{grad} \phi_{C} \cdot \operatorname{curl} \overline{\mathbf{w}}_{C}=0\end{cases}
$$

for each $\mathbf{w} \in X$, where $X$ is as in (9). Then $\mathbf{E}_{C}$ and $\mathbf{E}_{I}$ are determined in the same way as before. Let us also recall that, from (7), we have

$$
\int_{\Omega_{C}} \operatorname{grad} \phi_{C} \cdot \operatorname{curl} \mathbf{H}_{C}=\int_{\Gamma_{J}} \operatorname{curl} \mathbf{H}_{C} \cdot \mathbf{n} .
$$

Well-posedness of problem (12) comes from the theory of saddle-point problems. In fact, the sesquilinear form $\int_{\Omega_{C}} \boldsymbol{\sigma}^{-1} \operatorname{curl} \mathbf{H}_{C} \cdot \operatorname{curl} \overline{\mathbf{w}}_{C}+\int_{\Omega} i \omega \boldsymbol{\mu} \mathbf{H} \cdot \overline{\mathbf{w}}$ is coercive in $X$; moreover, since the unknown $V \in \mathbb{C}$ is a number, to show that the inf-sup condition is satisfied it is enough to find $\mathbf{w}^{*} \in X$ such that

$$
\left|\int_{\Omega_{C}} \operatorname{grad} \phi_{C} \cdot \operatorname{curl} \mathbf{w}_{C}^{*}\right|>0 .
$$

This can be done by taking the solution $\mathbf{w}_{C}^{*}$ to

$$
\begin{cases}\operatorname{curl} \mathbf{w}_{C}^{*}=\boldsymbol{\sigma} \operatorname{grad} \phi_{C} & \text { in } \Omega_{C} \\ \operatorname{div} \mathbf{w}_{C}^{*}=0 & \text { in } \Omega_{C} \\ \mathbf{w}_{C}^{*} \cdot \mathbf{n}=0 & \text { on } \Gamma_{E} \cup \Gamma_{J} \cup \Gamma,\end{cases}
$$


and the solution $\mathbf{w}_{I}^{*}$ to

$$
\begin{cases}\operatorname{curl} \mathbf{w}_{I}^{*}=\mathbf{0} & \text { in } \Omega_{I} \\ \operatorname{div} \mathbf{w}_{I}^{*}=0 & \text { in } \Omega_{I} \\ \mathbf{w}_{I}^{*} \times \mathbf{n}_{I}=-\mathbf{w}_{C}^{*} \times \mathbf{n}_{C} & \text { on } \Gamma \\ \mathbf{w}_{I}^{*} \cdot \mathbf{n}=0 & \text { on } \Gamma_{D} .\end{cases}
$$

Formulation (12) has been proposed also in [8] (for both Cases A and C). However, there it has been set $\mathbf{E}_{C}=\boldsymbol{\sigma}^{-1}$ curl $\mathbf{H}_{C}$, thus violating, for Case A, the Faraday equation on the surface $\Xi$.

- Electric ports: voltage excitation, E formulation

Having clarified that voltage excitation is equivalent to a source $V \boldsymbol{\sigma} \operatorname{grad} \phi_{C}$, the electric field formulation for Case A is easily devised: for each given $V \in \mathbb{C}$ find $\mathbf{E} \in Y$ such that

$$
\int_{\Omega} \boldsymbol{\mu}^{-1} \operatorname{curl} \mathbf{E} \cdot \operatorname{curl} \overline{\mathbf{z}}+\int_{\Omega_{C}} i \omega \boldsymbol{\sigma} \mathbf{E}_{C} \cdot \overline{\mathbf{z}}_{C}=-i \omega V \int_{\Omega_{C}} \boldsymbol{\sigma} \operatorname{grad} \phi_{C} \cdot \overline{\mathbf{z}}_{C}
$$

for each $\mathbf{z} \in Y$, where

$$
Y:=\left\{\mathbf{z} \in H(\operatorname{curl} ; \Omega) \mid \operatorname{div}\left(\boldsymbol{\epsilon}_{I} \mathbf{z}_{I}\right)=0 \text { in } \Omega_{I}, \mathbf{z} \times \mathbf{n}=\mathbf{0} \text { on } \partial \Omega\right\} .
$$

The existence of a solution $\mathbf{E}$ to problem (13) follows at once from what already proved for the $\mathbf{H}$ formulation. Uniqueness is straightforward.

The magnetic field $\mathbf{H}$ is then determined in $\Omega$ as $\mathbf{H}=-\frac{1}{i \omega} \boldsymbol{\mu}^{-1} \operatorname{curl} \mathbf{E}$.

A formulation similar to (13) (but based on the source term $V \boldsymbol{\sigma} \operatorname{grad} \Phi_{C}$, the function $\Phi_{C}$ having been defined in Remark 3.1) has been presented in [20]. However, there the electric field is not the solution to (13), but it is corrected, only in $\Omega_{C}$, by adding $V \operatorname{grad} \Phi_{C}$. Since it does not exist a curl-free vector field in $\Omega_{I}$ that has tangential component on $\Gamma$ equal to $V \operatorname{grad} \Phi_{C} \times \mathbf{n}$ (again, this is related to the solvability of problem (10)), this leads to the violation of the continuity of the tangential component of $\mathbf{E}$ through the interface $\Gamma$, thus to the violation of the Faraday law.

- Electric ports: current intensity excitation, $\mathrm{E}$ formulation

Since curl $\mathbf{H}_{C}=\boldsymbol{\sigma} \mathbf{E}_{C}+V \boldsymbol{\sigma} \operatorname{grad} \phi_{C}$, the variational formulation (for the sole Case A) now reads: for each given $I \in \mathbb{C}$, find $(\mathbf{E}, V) \in Y \times \mathbb{C}$ such that

$$
\left\{\begin{aligned}
& \int_{\Omega} \boldsymbol{\mu}^{-1} \operatorname{curl} \mathbf{E} \cdot \operatorname{curl} \overline{\mathbf{z}}+\int_{\Omega_{C}} i \omega \boldsymbol{\sigma} \mathbf{E}_{C} \cdot \overline{\mathbf{z}}_{C} \\
&+i \omega V \int_{\Omega_{C}} \boldsymbol{\sigma} \operatorname{grad} \phi_{C} \cdot \overline{\mathbf{z}}_{C}=0 \\
& \int_{\Omega_{C}} \operatorname{grad} \phi_{C} \cdot \boldsymbol{\sigma} \mathbf{E}_{C}+V \int_{\Omega_{C}} \boldsymbol{\sigma} \operatorname{grad} \phi_{C} \cdot \operatorname{grad} \phi_{C}=I
\end{aligned}\right.
$$

for each $\mathbf{z} \in Y$.

As before, existence of a solution is assured by the correspondent result for the magnetic field $\mathbf{H}$. Instead, uniqueness is a more delicate point. In fact, multiplying $(14)_{2}$ by $i \omega \bar{U}$, where $U \in \mathbb{C}$, we find

$$
\begin{aligned}
& \int_{\Omega} \boldsymbol{\mu}^{-1} \operatorname{curl} \mathbf{E} \cdot \operatorname{curl} \overline{\mathbf{z}} \\
& \quad+i \omega \int_{\Omega_{C}} \boldsymbol{\sigma}\left(\mathbf{E}_{C}+V \operatorname{grad} \phi_{C}\right) \cdot\left(\overline{\mathbf{z}}_{C}+\bar{U} \operatorname{grad} \phi_{C}\right)=i \omega I \bar{U} .
\end{aligned}
$$


Thus, putting $I=0$ and choosing $\mathbf{z}=\mathbf{E}$ and $U=V$, we obtain $\operatorname{curl} \mathbf{E}=\mathbf{0}$ in $\Omega$ and $\mathbf{E}_{C}+V \operatorname{grad} \phi_{C}=\mathbf{0}$ in $\Omega_{C}$. Since $\Omega$ is simply-connected, we also have $\mathbf{E}=\operatorname{grad} \psi$ in $\Omega$, and the boundary condition $\mathbf{E} \times \mathbf{n}=\mathbf{0}$ on $\partial \Omega$ gives $\psi=$ const. on $\partial \Omega$. Therefore, integrating $\mathbf{E}_{C}$ on the path $\widehat{\gamma}$ joining $\Gamma_{E}$ to $\Gamma_{J}$, we find

$$
\begin{aligned}
0 & =\int_{\widehat{\gamma}} \operatorname{grad} \psi_{C} \cdot d \boldsymbol{\tau}=\int_{\widehat{\gamma}} \mathbf{E}_{C} \cdot d \boldsymbol{\tau} \\
& =-\int_{\widehat{\gamma}} V \operatorname{grad} \phi_{C} \cdot d \boldsymbol{\tau}=-V .
\end{aligned}
$$

Thus $V=0$, and consequently $\mathbf{E}=\mathbf{0}$.

Also in this case, the magnetic field $\mathbf{H}$ is obtained in $\Omega$ by setting $\mathbf{H}=$ $-\frac{1}{i \omega} \boldsymbol{\mu}^{-1} \operatorname{curl} \mathbf{E}$.

Again, a formulation like (14) (but based on the source term $V \boldsymbol{\sigma} \operatorname{grad} \Phi_{C}$ ) has been proposed in [20]. The remark at the end of the preceding subsection still applies.

- Internal conductor: voltage excitation, $\mathbf{H}$ formulation

We have already made explicit the "voltage rule": applying a voltage is equivalent to consider a current density $\mathbf{J}_{e, C}=V \boldsymbol{\sigma} \boldsymbol{\rho}_{C}$. Then, for the $\mathbf{H}$-based formulation, Cases D and E can be studied as in [2]. Let us focus on Case F.

The problem reads: for each given $V \in \mathbb{C}$ find the unique solution $\mathbf{H} \in X$ to

$$
\int_{\Omega_{C}} \boldsymbol{\sigma}^{-1} \operatorname{curl} \mathbf{H}_{C} \cdot \operatorname{curl} \overline{\mathbf{w}}_{C}+\int_{\Omega} i \omega \boldsymbol{\mu} \mathbf{H} \cdot \overline{\mathbf{w}}=V \int_{\Omega_{C}} \boldsymbol{\rho}_{C} \cdot \operatorname{curl} \overline{\mathbf{w}}_{C}
$$

for each $\mathbf{w} \in X$, where

$$
X:=\left\{\mathbf{w} \in H(\operatorname{curl} ; \Omega) \mid \operatorname{curl} \mathbf{w}_{I}=\mathbf{0} \text { in } \Omega_{I}\right\} .
$$

Then set $\mathbf{E}_{C}:=\boldsymbol{\sigma}^{-1} \operatorname{curl} \mathbf{H}_{C}-V \boldsymbol{\rho}_{C}$ in $\Omega_{C}$, and in $\Omega_{I}$ define $\mathbf{E}_{I}$ to be the solution to

$$
\begin{cases}\operatorname{curl} \mathbf{E}_{I}=-i \omega \boldsymbol{\mu}_{I} \mathbf{H}_{I} & \text { in } \Omega_{I} \\ \operatorname{div}\left(\boldsymbol{\epsilon}_{I} \mathbf{E}_{I}\right)=0 & \text { in } \Omega_{I} \\ \mathbf{E}_{I} \times \mathbf{n}_{I}=-\mathbf{E}_{C} \times \mathbf{n}_{C} & \text { on } \Gamma \\ \boldsymbol{\epsilon}_{I} \mathbf{E}_{I} \cdot \mathbf{n}=0 & \text { on } \partial \Omega\end{cases}
$$

Again, the main problem here is the solvability of (16). For the internal conductor, this has been already done in [2], to which we refer.

Let us also recall that the same variational formulation (15) has been proposed in [17], [25] and [20]. However, there it has been set $\mathbf{E}_{C}:=\boldsymbol{\sigma}^{-1} \operatorname{curl} \mathbf{H}_{C}$, leading to the violation of the Faraday equation on the surface $\Lambda$ cutting the basic non-bounding cycle of $\Omega_{I}$.

- Internal conductor: current intensity excitation, $\mathbf{H}$ formulation

Let us start focusing on Case F. Recalling (8), the problem is: for each given $I \in \mathbb{C}$ find the unique solution $(\mathbf{H}, V) \in X \times \mathbb{C}$ to

$$
\begin{cases}\int_{\Omega_{C}} \boldsymbol{\sigma}^{-1} \operatorname{curl} \mathbf{H}_{C} \cdot \operatorname{curl} \overline{\mathbf{w}}_{C} & +\int_{\Omega} i \omega \boldsymbol{\mu} \mathbf{H} \cdot \overline{\mathbf{w}} \\ \int_{\Omega_{C}} \boldsymbol{\rho}_{C} \cdot \operatorname{curl} \mathbf{H}_{C}=I & -V \int_{\Omega_{C}} \boldsymbol{\rho}_{C} \cdot \operatorname{curl} \overline{\mathbf{w}}_{C}=0\end{cases}
$$


for each $\mathbf{w} \in X$, where $X$ is as in (15); then set $\mathbf{E}_{C}:=\sigma^{-1} \operatorname{curl} \mathbf{H}_{C}-V \boldsymbol{\rho}_{C}$ in $\Omega_{C}$ and determine $\mathbf{E}_{I}$ as in (16).

Well-posedness of problem (17) comes from the theory of saddle-point problems. Taking into account what we have already presented for the electric port case, it is enough to find $\mathbf{w}^{*} \in X$ such that

$$
\left|\int_{\Omega_{C}} \rho_{C} \cdot \operatorname{curl} \mathbf{w}_{C}^{*}\right|>0 \text {. }
$$

This can be done by taking the solution $\mathbf{w}_{C}^{*}$ to

$$
\begin{cases}\operatorname{curl} \mathbf{w}_{C}^{*}=\boldsymbol{\sigma} \boldsymbol{\rho}_{C} & \text { in } \Omega_{C} \\ \operatorname{div} \mathbf{w}_{C}^{*}=0 & \text { in } \Omega_{C} \\ \mathbf{w}_{C}^{*} \times \mathbf{n}_{C}=c_{0} \boldsymbol{\rho}_{I} \times \mathbf{n}_{C} & \text { on } \Gamma,\end{cases}
$$

and the solution $\mathbf{w}_{I}^{*}$ to

$$
\begin{cases}\operatorname{curl} \mathbf{w}_{I}^{*}=\mathbf{0} & \text { in } \Omega_{I} \\ \operatorname{div} \mathbf{w}_{I}^{*}=0 & \text { in } \Omega_{I} \\ \mathbf{w}_{I}^{*} \times \mathbf{n}_{I}=c_{0} \boldsymbol{\rho}_{I} \times \mathbf{n}_{I} & \text { on } \Gamma \\ \mathbf{w}_{I}^{*} \times \mathbf{n}=\mathbf{0} & \text { on } \partial \Omega \\ \int_{\partial \Omega} \mathbf{w}_{I}^{*} \cdot \mathbf{n}=0, & \end{cases}
$$

where $c_{0}=\int_{\Omega_{C}} \boldsymbol{\sigma} \boldsymbol{\rho}_{C} \cdot \boldsymbol{\rho}_{C}$. Here $\boldsymbol{\rho}_{I}$ is the basis function of the space

$$
\mathcal{H}\left(\Omega_{I}\right):=\left\{\boldsymbol{\eta} \in\left(L^{2}\left(\Omega_{I}\right)\right)^{3} \mid \operatorname{curl} \boldsymbol{\eta}=\mathbf{0}, \operatorname{div} \boldsymbol{\eta}=0, \boldsymbol{\eta} \cdot \mathbf{n}=0 \text { on } \partial \Omega \cup \Gamma\right\},
$$

normalized by $\int_{\widetilde{\gamma}} \rho_{I} \cdot d \boldsymbol{\tau}=1$, where $\widetilde{\gamma}$ is the basic non-bounding cycle entering in the "handle" of $\Omega_{C}$, and oriented consistently with the non-bounding cycle $\gamma$ which runs in $\Omega_{C}$ (namely, each one is oriented counterclockwise with respect to the other). Note that the existence of the solution $\mathbf{w}_{C}^{*}$ is a consequence of the relation $\int_{\Gamma}\left(\boldsymbol{\rho}_{C} \times \mathbf{n}_{C}\right) \cdot \boldsymbol{\rho}_{I}=1$.

To complete the presentation, let us note that, if interested in considering Case $\mathrm{D}$, one has to substitute in (16) the boundary condition $\boldsymbol{\epsilon}_{I} \mathbf{E}_{I} \cdot \mathbf{n}=0$ on $\partial \Omega$ with $\mathbf{E}_{I} \times \mathbf{n}=\mathbf{0}$ on $\partial \Omega$ and add the condition $\int_{\partial \Omega} \boldsymbol{\epsilon}_{I} \mathbf{E}_{I} \cdot \mathbf{n}=0$.

Instead, concerning Case $\mathrm{E}$, one has to use in (17) the space

$$
X:=\left\{\mathbf{w} \in H(\operatorname{curl} ; \Omega) \mid \operatorname{curl} \mathbf{w}_{I}=\mathbf{0} \text { in } \Omega_{I}, \mathbf{w}_{I} \times \mathbf{n}=\mathbf{0} \text { on } \partial \Omega\right\} .
$$

\section{- Internal conductor: voltage excitation, E formulation}

We are not going to give details for this case. In fact, the "voltage rule" is telling us that we have just to consider a current density $\mathbf{J}_{e, C}=V \boldsymbol{\sigma} \boldsymbol{\rho}_{C}$, hence this formulation is easily devised (for instance, for Cases $\mathrm{D}$ and $\mathrm{E}$ one can follow what done in [4]). Moreover, the case in which excitation is due to the current intensity can also illustrate the functional framework to be used for the voltage excitation case (in this respect, see the first equation in (18)). 
- Internal conductor: current intensity excitation, $\mathrm{E}$ formulation The "current intensity rule" says that the given current intensity $I$ is generating not only the electric field but also a current density $V \boldsymbol{\sigma} \boldsymbol{\rho}_{C}$. Moreover, we have curl $\mathbf{H}_{C}=\boldsymbol{\sigma} \mathbf{E}_{C}+V \boldsymbol{\sigma} \rho_{C}$. Then, the problem is: for each given $I \in \mathbb{C}$ find $(\mathbf{E}, V) \in Y \times \mathbb{C}$ such that

$$
\left\{\begin{array}{c}
\int_{\Omega} \boldsymbol{\mu}^{-1} \operatorname{curl} \mathbf{E} \cdot \operatorname{curl} \overline{\mathbf{z}}+\int_{\Omega_{C}} i \omega \boldsymbol{\sigma} \mathbf{E}_{C} \cdot \overline{\mathbf{z}}_{C} \\
\quad+i \omega V \int_{\Omega_{C}} \boldsymbol{\sigma} \boldsymbol{\rho}_{C} \cdot \overline{\mathbf{z}}_{C}=0 \\
\int_{\Omega_{C}} \boldsymbol{\rho}_{C} \cdot \boldsymbol{\sigma} \mathbf{E}_{C}+V \int_{\Omega_{C}} \boldsymbol{\sigma} \boldsymbol{\rho}_{C} \cdot \boldsymbol{\rho}_{C}=I
\end{array}\right.
$$

for each $\mathbf{z} \in Y$, where

$$
Y:=\left\{\begin{array}{cc}
\left\{\mathbf{z} \in H(\operatorname{curl} ; \Omega) \mid \operatorname{div}\left(\boldsymbol{\epsilon}_{I} \mathbf{z}_{I}\right)=0 \text { in } \Omega_{I},\right. & \\
\left.\quad \mathbf{z} \times \mathbf{n}=\mathbf{0} \text { on } \partial \Omega, \int_{\partial \Omega} \boldsymbol{\epsilon}_{I} \mathbf{E}_{I} \cdot \mathbf{n}=0\right\} & \text { for Case D } \\
\left\{\mathbf{z} \in H(\operatorname{curl} ; \Omega) \mid \operatorname{div}\left(\boldsymbol{\epsilon}_{I} \mathbf{z}_{I}\right)=0 \text { in } \Omega_{I},\right. & \text { for Case E } \\
\left.\boldsymbol{\epsilon}_{I} \mathbf{z}_{I} \cdot \mathbf{n}=0 \text { on } \partial \Omega\right\} & \\
\left\{\mathbf{z} \in H(\operatorname{curl} ; \Omega) \mid \operatorname{div}\left(\boldsymbol{\epsilon}_{I} \mathbf{z}_{I}\right)=0 \text { in } \Omega_{I},\right. & \text { for Case F. }
\end{array}\right.
$$

As before, existence is a consequence of what already proved for the $\mathbf{H}$ formulation. Concerning uniqueness, by proceeding as in the electric port case we find curl $\mathbf{E}=\mathbf{0}$ in $\Omega$ and $\mathbf{E}_{C}=-V \boldsymbol{\rho}_{C}$ in $\Omega_{C}$. Since $\Omega$ is simply-connected, we also have $\mathbf{E}=\operatorname{grad} \psi$ in $\Omega$. Therefore, integrating $\mathbf{E}_{C}$ on the cycle $\gamma$ we find

$$
\begin{aligned}
0 & =\int_{\gamma} \operatorname{grad} \psi_{C} \cdot d \boldsymbol{\tau}=\int_{\gamma} \mathbf{E}_{C} \cdot d \boldsymbol{\tau} \\
& =-\int_{\gamma} V \boldsymbol{\rho}_{C} \cdot d \boldsymbol{\tau}=-V .
\end{aligned}
$$

Thus $V=0$, and consequently $\mathbf{E}=\mathbf{0}$.

Having solved (18), the magnetic field in $\Omega$ is as usual defined as $\mathbf{H}=$ $-\frac{1}{i \omega} \boldsymbol{\mu}^{-1} \operatorname{curl} \mathbf{E}$.

A similar formulation has been proposed in [20], [6] (in the former paper, replacing the source $V \boldsymbol{\rho}_{C}$ by $V \operatorname{grad} \widetilde{\Phi}_{C}, \widetilde{\Phi}_{C}$ being a function jumping by 1 through a section $\Sigma$ of $\Omega_{C}$ ). However, in these papers the electric field is not the solution $\mathbf{E}_{C}$ to $(18)_{1}$, but it is corrected in $\Omega_{C}$ by adding the source term. In this way Faraday law is no more verified across the interface $\Gamma$.

The same remark applies for the voltage excitation problem of the preceding subsection and the formulations proposed in [18], [20].

\section{Numerical approximation}

The variational formulations presented in the preceding section can be used as a starting point for devising finite element methods for approximating the solution.

In fact, the voltage excitation reduces to a standard problem with a given current density $\left(V \boldsymbol{\sigma} \operatorname{grad} \phi_{C}\right.$ or else $\left.V \boldsymbol{\sigma} \boldsymbol{\rho}_{C}\right)$, therefore any method used for eddy-current problems can be applied: without any attempt of being complete, 
let us only mention those proposed in [15], [7], [1], [3] for the $\mathbf{H}$-formulation, and in [24], [9], [21], [22], [4] for the $\mathbf{E}$-formulation (or for the related magnetic vector potential formulation).

It is worthy to note that, when considering the $\mathbf{H}$-formulation, it is not needed to construct the functions $\operatorname{grad} \phi_{C}$ or $\boldsymbol{\rho}_{C}$. In fact, to give an example for the electric port case, one can proceed in this way: consider a fixed (and coarse) mesh in $\Omega_{C}$, and let $\mathcal{I}_{*}^{C}$ be the finite element interpolant taking value 0 everywhere, except on $\Gamma_{J}$, where it has value 1 . Then define $\phi_{*}$ to be the solution to

$$
\begin{cases}\operatorname{div}\left(\boldsymbol{\sigma} \operatorname{grad} \phi_{*}\right)=-\operatorname{div}\left(\boldsymbol{\sigma} \operatorname{grad} \mathcal{I}_{*}^{C}\right) & \text { in } \Omega_{C} \\ \phi_{*}=0 & \text { on } \Gamma_{E} \cup \Gamma_{J} \\ \boldsymbol{\sigma} \operatorname{grad} \phi_{*} \cdot \mathbf{n}=-\boldsymbol{\sigma} \operatorname{grad} \mathcal{I}_{*}^{C} \cdot \mathbf{n} & \text { on } \Gamma .\end{cases}
$$

Thus $\phi_{C}=\mathcal{I}_{*}^{C}+\phi_{*}$ in $\Omega_{C}$, and

$$
\begin{aligned}
\int_{\Omega_{C}} \operatorname{grad} \phi_{C} \cdot \operatorname{curl} \overline{\mathbf{w}}_{C} & =\int_{\Omega_{C}}\left(\operatorname{grad} \mathcal{I}_{*}^{C}+\operatorname{grad} \phi_{*}\right) \cdot \operatorname{curl} \overline{\mathbf{w}}_{C} \\
& =\int_{\Omega_{C}} \operatorname{grad} \mathcal{I}_{*}^{C} \cdot \operatorname{curl} \overline{\mathbf{w}}_{C},
\end{aligned}
$$

as div curl $\overline{\mathbf{w}}_{C}=0, \phi_{*}=0$ on $\Gamma_{E} \cup \Gamma_{J}$ and $\operatorname{curl} \overline{\mathbf{w}}_{C} \cdot \mathbf{n}=0$ on $\Gamma$.

Therefore, we have verified that in the $\mathbf{H}$-based variational formulations one can substitute $\phi_{C}$ by the easily computable $\mathcal{I}_{*}^{C}$, and the solution $\mathbf{H}$ remains the same. Clearly, the need to compute $\phi_{C}$ (namely, $\phi_{*}$ ) comes again into play if one wants to recover $\mathbf{E}_{C}$, which is given by

$$
\mathbf{E}_{C}=\boldsymbol{\sigma}^{-1} \operatorname{curl} \mathbf{H}_{C}-V \operatorname{grad} \phi_{C}=\boldsymbol{\sigma}^{-1} \operatorname{curl} \mathbf{H}_{C}-V \operatorname{grad} \mathcal{I}_{*}^{C}-V \operatorname{grad} \phi_{*} .
$$

If the current intensity is given, the constraint $\int_{\Omega_{C}} \mathbf{Q}_{C} \cdot \operatorname{curl} \mathbf{H}_{C}=I$ has to be added (here $\mathbf{Q}_{C}=\operatorname{grad} \phi_{C}$ or else $\mathbf{Q}_{C}=\boldsymbol{\rho}_{C}$ ). In the $\mathbf{H}$-formulation, the voltage $V$ plays the role of a Lagrange multiplier associated to this constraint, and the global problem is a saddle-point problem. For any type of conforming finite element discretization using edge elements in $\Omega_{C}$, the presence of this Lagrange multiplier requires that an inf-sup condition like

$$
\left|\int_{\Omega_{C}} \mathbf{Q}_{C} \cdot \operatorname{curl} \mathbf{w}_{C, h}^{*}\right| \geq \beta|| \mathbf{w}_{h}^{*} \|_{X}
$$

is satisfied for a constant $\beta>0$, independent of $h$, and a suitable discrete vector function $\mathbf{w}_{h}^{*}$.

This can be done as follows (for instance, let us focus on the electric port case): expressing $\operatorname{grad} \phi_{C}$ in terms of $\operatorname{grad} \mathcal{I}_{*}^{C}$, as done before, we have by integration by parts and the Stokes theorem

$$
\begin{aligned}
\int_{\Omega_{C}} \operatorname{grad} \phi_{C} \cdot \operatorname{curl} \mathbf{w}_{C, h}^{*} & =\int_{\Omega_{C}} \operatorname{grad} \mathcal{I}_{*}^{C} \cdot \operatorname{curl} \mathbf{w}_{C, h}^{*}=\int_{\Gamma_{J}} \operatorname{curl} \mathbf{w}_{C, h}^{*} \cdot \mathbf{n} \\
& =\int_{\partial \Gamma_{J}} \mathbf{w}_{C, h}^{*} \cdot d \boldsymbol{\tau}=\int_{\partial \Gamma_{J}} \mathbf{w}_{I, h}^{*} \cdot d \boldsymbol{\tau} .
\end{aligned}
$$

Let us consider a fixed (and coarse) mesh in $\Omega$, and let $\mathcal{I}_{*}^{I}$ be the finite element interpolant taking value 0 everywhere in $\Omega_{I}$, except on the cutting surface $\Xi$, 
transversal to $\Gamma_{D}$ and $\Gamma$, where it has a double value, 0 on one side and 1 on the other side (following the orientation of $\partial \Gamma_{J}$, that is counterclockwise with respect to $\mathbf{n}$ on $\Gamma_{J}$ ). From now on we consider triangulations that are all obtained as a refinement of the basic coarse mesh, in such a way that a discrete function on the coarse mesh is also a discrete function on all the other meshes. Then choose as $\mathbf{w}_{I, h}^{*}$ the $\left(L^{2}\left(\Omega_{I}\right)\right)^{3}$-extension of $\operatorname{grad} \mathcal{I}_{*}^{I}$, computed in $\Omega_{I} \backslash \Xi$; note that $\operatorname{grad} \mathcal{I}_{*}^{I} \times \mathbf{n}$ is defined in a unique way on $\Xi$, as the jump of $\mathcal{I}_{*}^{I}$ on $\Xi$ is equal to 1 . Finally, take as $\mathbf{w}_{C, h}^{*}$ the edge element interpolant, on the coarse mesh in $\Omega_{C}$, of the value $\mathbf{w}_{I, h}^{*} \times \mathbf{n}_{I}$ on $\Gamma$. It is easily checked that with this choice $\int_{\partial \Gamma_{J}} \mathbf{w}_{I, h}^{*} \cdot d \boldsymbol{\tau}=1$ and that the norm $\left\|\mathbf{w}_{h}^{*}\right\|_{X}$ does not depend on $h$, therefore the inf-sup condition is satisfied.

Coming to the E-formulation, when the current intensity is assigned it takes a non-standard form: in fact, in (14) and (18) it is questionable that the sesquilinear forms at the left hand sides are coercive, and, on the other hand, the current intensity condition is not a pure constraint, so that these problems are not saddle-point problems. In this paper we have proved existence and uniqueness of the solution for the infinite dimensional case, but a complete analysis of a finite element approximation method could be a more delicate point. However, this approach has been used in [6] for an axisymmetric problem, with good numerical performances.

Remark 5.1. When the current intensity is assigned, it is possible to devise an alternative formulation in terms of a magnetic vector potential and an electric scalar potential, with the Coulomb gauge. Namely, one looks for $\mathbf{A}$ and $v_{C}$ such that

$$
\boldsymbol{\mu} \mathbf{H}=\operatorname{curl} \mathbf{A} \quad \text { in } \Omega, \mathbf{E}_{C}=-i \omega \mathbf{A}_{C}-\operatorname{grad} v_{C} \quad \text { in } \Omega_{C},
$$

with $\operatorname{div} \mathbf{A}=0$ in $\Omega$ and $\mathbf{A} \cdot \mathbf{n}=0$ on $\partial \Omega$.

Writing (14) and (18) in terms of these unknowns, and inserting the gauging term as a penalization, as usually done with this approach, one ends up with a sesquilinear form that can be proved to be coercive (for similar computations see [12], where the analysis of the $\mathbf{A}-v_{C}$ method is presented when the excitation is due to a given current density $\mathbf{J}_{e}$ ).

Acknowledgement. It is a pleasure to thank Oszkár Bíró and Rafael Vázquez for some useful conversations about the subject of this paper.

\section{References}

[1] A. Alonso Rodríguez, P. Fernandes, And A. Valli, The timeharmonic eddy-current problem in general domains: solvability via scalar potentials, in Computational Electromagnetics, C. Carstensen, S. Funken, W. Hackbusch, R. Hoppe, and P. Monk, eds., Springer, Berlin, 2003, pp. 143-163. 
[2] — Weak and strong formulations for the time-harmonic eddy-current problem in general multi-connected domains, European J. Appl. Math., 14 (2003), pp. 387-406.

[3] A. Alonso Rodríguez, R. Hiptmair, and A. Valli, Mixed finite element approximation of eddy current problems, IMA J. Numer. Anal., 24 (2004), pp. 255-271.

[4] A. Alonso Rodríguez And A. Valli, Mixed finite element approximation of eddy current problems based on the electric field, in ECCOMAS 2004: European Congress on Computational Methods in Applied Sciences and Engineering, P. Neittaanmäki, T. Rossi, K. Majava, and O. Pironneau, eds., University of Jyvs̈kylä, Jyväskylä, 2004. http://www.mit.jyu.fi/eccomas2004/proceedings/pdf/185.pdf.

[5] A. Alonso Rodríguez, A. Valli, and R. Vázquez, A formulation of the eddy-current problem in the presence of electric ports, Report UTM, Department of Mathematics, University of Trento, 2007.

[6] A. Bermúdez, D. Gómez, M. C. Muñzz, and P. Salgado, A FEM/BEM for axisymmetric electromagnetic and thermal modelling of induction furnaces, Int. J. Numer. Meth. Engng., (2007). To appear.

[7] A. Bermúdez, R. Rodríguez, and P. Salgado, A finite element method with Lagrange multipliers for low-frequency harmonic Maxwell equations, SIAM J. Numer. Anal., 40 (2002), pp. 1823-1849.

[8] - Numerical solution of eddy-current problems in bounded domains using realistic boundary conditions, Comput. Methods Appl. Mech. Engrg., 194 (2005), pp. 411-426.

[9] O. Bíró And K. Preis, On the use of magnetic vector potential in the finite element analysis of three-dimensional eddy currents, IEEE Trans. Magn., 25 (1989), pp. 3145-3159.

[10] O. Bíró, K. Preis, G. Buchgraber, And I. TičAR, Voltage-driven coils in finite-element formulations using a current vector and a magnetic scalar potential, IEEE Trans. Magn., 40 (2004), pp. 1286-1289.

[11] O. Bíró, K. Preis, W. Renhart, G. Vrisk, and K. R. Richter, Computation of 3-D current driven skin effect problems using a current vector potential, IEEE Trans. Magn., 29 (1993), pp. 1325-1328.

[12] O. Bíro AND A. VALLI, The Coulomb gauged vector potential formulation for the eddy-current problem in general geometry: well-posedness and numerical approximation, Comput. Methods Appl. Mech. Engrg., 196 (2007), pp. $1890-1904$.

[13] A. Bossavit, Computational Electromagnetism. Variational Formulation, Complementarity, Edge Elements, Academic Press, San Diego, 1998. 
[14] —, Most general 'non-local' boundary conditions for the Maxwell equations in a bounded region, COMPEL, 19 (2000), pp. 239-245.

[15] A. Bossavit and J. C. VÉrité, A mixed FEM/BIEM method to solve eddy-current problems, IEEE Trans. Magn., MAG-18 (1982), pp. 431-435.

[16] P. DulaR, The benefits of nodal and edge elements coupling for discretizing global constraints in dual magnetodynamic formulations, J. Comput. Appl. Math., 168 (2004), pp. 165-178.

[17] P. Dular, C. Geuzaine, and W. Legros, A natural method for coupling magnetodynamic $\mathbf{H}$-formulations and circuits equations, IEEE Trans. Magn., 35 (1999), pp. 1626-1629.

[18] P. Dular, F. Henrotte, And W. Legros, A general and natural method to define circuits relations associated with magnetic vector potential formulations, IEEE Trans. Magn., 35 (1999), pp. 1630-1633.

[19] P. Dular, W. Legros, And A. Nicolet, Coupling of local and global quantities in various finite element formulations and its application to electrostatics, magnetostatics and magnetodynamics, IEEE Trans. Magn., 34 (1998), pp. 3078-3081.

[20] R. Hiptmair and O. Sterz, Current and voltage excitations for the eddy current model, Int. J. Numer. Modelling, 18 (2005), pp. 1-21.

[21] H. Kanayama and F. KikUChi, 3-D eddy current computation using Nedelec elements, Information, 2 (1999), pp. 37-45.

[22] H. Kanayama, D. Tagami, M. Saito, and F. Kikuchi, A numerical method for 3-D eddy current problems, Japan J. Indust. Appl. Math., 18 (2001), pp. 603-612.

[23] G. Meunier, Y. Le Floch, And C. Guérin, A nonlinear circuit coupled $\mathbf{t}-\mathbf{t}_{\mathbf{0}}-\phi$ formulation for solid conductors, IEEE Trans. Magn., 39 (2003), pp. 1729-1732.

[24] T. MoRisue, Magnetic vector potential and electric scalar potential in three-dimensional eddy current problem, IEEE Trans. Magn., MAG-18 (1982), pp. 531-535.

[25] J. Rappaz, M. Swierkosz, and C. Trophime, Un modèle mathématique et numérique pour un logiciel de simulation tridimensionnelle d'induction électromagnétique, Tech. Rep. 05.99, Département de Mathématiques, École Polytechnique Fédérale de Lausanne, 1999.

[26] G. Rubinacci, A. Tamburrino, and F. Villone, Circuits/fields coupling and multiply connected domains in integral formulations, IEEE Trans. Magn., 38 (2002), pp. 581-584. 